\title{
BMJ Open Exploring variation in how ambulance services address non-conveyance: a qualitative interview study
}

\author{
Emma Knowles, Lindsey Bishop-Edwards, Alicia O'Cathain
}

To cite: Knowles E, BishopEdwards L, O'Cathain A. Exploring variation in how ambulance services address non-conveyance: a qualitative interview study. BMJ Open 2018;8:e024228. doi:10.1136/ bmjopen-2018-024228

- Prepublication history for this paper is available online. To view these files, please visit the journal online (http://dx.doi. org/10.1136/bmjopen-2018024228).

Received 17 May 2018 Revised 4 October 2018 Accepted 16 October 2018

D Check for updates

(C) Author(s) (or their employer(s)) 2018. Re-use permitted under CC BY-NC. No commercial re-use. See rights and permissions. Published by BMJ.

School of Health and Related Research (ScHARR), University of Sheffield, Sheffield, UK

Correspondence to

Emma Knowles;

e.l.knowles@sheffield.ac.uk

\begin{abstract}
Objectives There is considerable variation in nonconveyance rates between ambulance services in England. The aim was to explore variation in how each ambulance service addressed non-conveyance for calls ending in telephone advice and discharge at scene.

Design A qualitative interview study.

Setting Ten large regional ambulance services covering $99 \%$ of the population in England.
\end{abstract}

Participants Between four and seven interviewees from each ambulance service including managers, paramedics and healthcare commissioners, totalling 49 interviews.

Methods Telephone semistructured interviews.

Results The way interviewees in each ambulance service discussed non-conveyance within their organisation varied for three broad themes. First, ambulance service senior management appeared to set the culture around non-conveyance within an organisation, viewing it either as an opportunity or as a risky endeavour. Although motivation levels to undertake non-conveyance did not appear to be directly affected by the stability of an ambulance service in terms of continuity of leadership and externally assessed quality, this stability could affect the ability of the organisation to innovate to increase non-conveyance rates. Second, descriptions of workforce configuration differed between ambulance services, as well as how this workforce was used, trained and valued. Third, interviewees in each ambulance service described health and social care in the wider emergency and urgent care system differently in terms of availability of services that could facilitate non-conveyance, the amount of collaborative working between health and social care services and the ambulance service and complexity related to the numbers of services and healthcare commissioners with whom they had to work.

Conclusions This study suggests that factors within and outside the control of ambulance services may contribute to variation in non-conveyance rates. These findings can be tested in a quantitative analysis of factors affecting variation in non-conveyance rates between ambulance services in England.

\section{BACKGROUND}

Emergency ambulance services, known as Emergency Medical Services in some countries, deal with significant numbers of calls each year. In England in 2016/2017, ambulance services responded to 7 million calls for

\section{Strengths and limitations of this study}

This was a national study of ambulance services covering $99 \%$ of the population in England.

- It is the first study of organisational factors potentially affecting variation in non-conveyance rates.

- A breadth of perspectives was captured from paramedics through to senior managers within each ambulance service.

- The small number of interviews in each service may not captured the diversity of views within each service.

- Paramedic interviewees were selected by managers and may have been selected because they concurred with the managerial view of non-conveyance.

a population of around 55 million. ${ }^{1}$ When a call is received by an ambulance service in England, it can be dealt with in a number of ways: some patients receive clinical assessment over the telephone without the need for an ambulance, some patients are sent an ambulance and then discharged at scene and some patients are sent an ambulance and conveyed to an emergency department or other healthcare service such as a walk-in centre. Non-conveyance rates, where patients are not transported to a hospital, are high in England compared with other countries. The rate in England in 2016/2017 of patients not conveyed to a hospital with an emergency department was $47 \%^{1}$ compared with rates of $42 \%$ in Finland ${ }^{2}$ and $18 \%$ in Canada. ${ }^{3}$ This international variation in non-conveyance rates for ambulance services has existed for decades. ${ }^{4}$

Although some non-conveyance is caused by patient refusal to travel, health policy in England promotes the use of non-conveyance by ambulance services as a way of offering care closer to home. ${ }^{5}$ As well as offering the most appropriate response to patients' needs, non-conveyance can help the efficiency of the emergency and urgent care system in which ambulance services operate. For example, high rates of non-conveyance by ambulance 
services have been associated with lower rates of avoidable emergency admissions. ${ }^{6}$

The importance of non-conveyance for English ambulance services is evident in the use of four national quality indicators measuring rates of different types of non-conveyance as part of the publically available Ambulance Quality Indicators. ${ }^{1}$ These indicators show considerable variation in non-conveyance rates between the regional ambulance services in England. In February 2018, for the 10 regional ambulance services covering $99 \%$ of the population of England, there were 633772 incidents. Across the services, rates of incidents ending in telephone advice varied between $4 \%$ and $8 \%$ and variation in discharge at scene rates varied between $23 \%$ and $37 \%$.

Patient characteristics can determine the response offered by an ambulance service. For example, a recent systematic review concluded that patients who are sent an ambulance and discharged at scene are more likely to be older and more likely to have fallen. ${ }^{7}$ However, little is known about ambulance service characteristics that may affect non-conveyance rates in terms of both the characteristics of the organisations and the emergency and urgent care systems in which they operate. As part of a wider mixed methods study on explaining Variation in Ambulance Non-conveyance rates (the VAN project), a qualitative interview study was undertaken with staff within ambulance services and healthcare commissioners of each ambulance service. ${ }^{8}$ The aim of the qualitative study was to explore how each ambulance service addressed non-conveyance, with regard to telephone advice and discharge at scene, in order to identify potential factors affecting variation between ambulance services.

\section{METHODS}

\section{Setting and context}

In England, UK Emergency Medical Services are provided by 11 ambulance services within the National Health Service (NHS). Ten of these ambulance services deal with over 99\% of emergency ambulance calls from the population of England. Most calls are from patients calling 999 to request an ambulance-approximately four in five calls-with the remaining calls being passed through from patients calling a telephone helpline for urgent care called 'NHS 111', or through direct referral from a health professional requesting an emergency ambulance. Calls are taken by non-clinical staff who use software to identify the priority of each call. The 11 ambulance services use two different priority dispatch systems: Medical Priority Dispatch System and NHS Pathways. A small percentage of calls that are categorised as low priority are passed to clinicians in Emergency Operating Centres or Clinical Hubs within each ambulance service for secondary triage. The clinicians in these Emergency Operating Centres or Hubs use a decision support software to determine whether the patient needs an ambulance or can be offered telephone advice only. This advice includes self-care advice or referral to a service such as primary care; an ambulance is not dispatched. For calls not sent for secondary triage, an ambulance is dispatched and patients may be attended by paramedics, paramedics with extended skills, or emergency care technicians with basic emergency training. The ambulance crew assesses and treats patients at scene and can discharge people at scene, transport them to a hospital-based emergency department or transport them to another health facility such as a walk-in centre. Decisions are made by paramedics in conjunction with patients and their families. Protocols exist for making decisions relating to transport of patients with some health conditions. Sometimes non-transport occurs due to patient refusal to travel. Health services within the NHS in England are commissioned by around 200 Clinical Commissioning Groups ${ }^{9}$ that hold the budget for ensuring services are available for their geographically-based populations. Each regional ambulance service has a lead commissioner from the set of Clinical Commissioning Groups commissioning services for the populations served by that ambulance service.

\section{The wider mixed methods study}

The VAN project included a sequential mixed methods study aiming to explain variation in non-conveyance rates between ambulance services in England. ${ }^{8}$ The 10 larger ambulance services in England were included in the study. The 11th service was excluded because of its relatively small size. The first phase was a qualitative interview study to identify potential factors which may contribute to variation in non-conveyance rates. The second phase was a statistical regression to determine the amount of variation these factors explained in non-conveyance rates between the 10 large regional ambulance services. The first phase only is reported in this article.

\section{The qualitative interview study}

A telephone interview study was undertaken with three groups within each of the 10 ambulance services: ambulance service managers, paramedics and healthcare commissioners of the ambulance service. The aim was to understand their perceptions of influences on non-conveyance rates within their regional ambulance service. These three groups were selected because: ambulance service managers can shape strategic decisions that affect non-conveyance rates at an organisational level, such as workforce configuration or investment in staff training; paramedics make daily decisions about whether to convey patients to hospital or not and can offer views of patient characteristics, workforce practices and organisational factors affecting non-conveyance; and healthcare commissioners can encourage ambulance services to undertake non-conveyance through setting targets for non-conveyance rates within contracts and offering additional investment for non-conveyance initiatives. The plan was to undertake approximately 50 interviews: interviews with two managers (one at director level and one in operational management), two paramedics (a paramedic with extended skills and one without), and the lead healthcare commissioner for each of the ten large regional ambulance services in England. 
Interviewees were recruited by working with a local collaborator based in each ambulance service. Typically, these were a Research and Development manager or an operational manager with responsibility for non-conveyance. Healthcare commissioners leading on commissioning ambulance services in each region were identified by approaching the ambulance service or Clinical Commissioning Groups. One researcher (LB-E) undertook all the interviews by telephone. The researcher had no specialist knowledge of, or prior relationship with, the ambulance service. Interviews took place at an individual, rather than group, level. The researcher was blind to the non-conveyance rates of the interviewees' organisations. The interviews were semi-structured. A topic guide was used, covering perceptions of factors that affected non-conveyance within that service. The topic guide was informed by the evidence base as well as the study research questions. For example, paramedics with extended skills are known to undertake more non-conveyance than other paramedics ${ }^{10}$ so workforce was included as a prompt within the topic guide. The topic guide was piloted within the first few interviews (included in the analysis) and only minor changes were required. Interviews were digitally audio recorded and lasted between 40 and $90 \mathrm{~min}$. The majority of interviews were conducted in 2015 , with a small number taking place early in 2016.

\section{Analysis}

The digital recordings of interviews were transcribed verbatim. Transcripts were checked, alongside recordings, for accuracy prior to uploading to the qualitative software package Nvivo V.10 for analysis. ${ }^{11}$ Transcripts were not returned to participants for comment. Data were analysed using the framework approach. ${ }^{12}$ A small number of transcripts from a variety of types of interviewee were read by EK, LB-E and AO'C for familiarisation. A thematic framework was constructed based on the topic guide and emergent themes from reading the transcripts. The initial framework was developed by EK and revised following discussions with AO'C and LB-E. The thematic framework included: views of non-conveyance rates, calculation of rates, national drivers, organisational drivers, collaborative working within the emergency and urgent care system, workforce issues (skill mix, culture, working patterns), triage software, patient characteristics, defining and measuring appropriate non-conveyance, and commissioning non-conveyance. Discussions specifically relating to telephone advice only or discharge at scene were coded in addition to discussion of non-conveyance generally. Each transcript was coded to the themes and subthemes, by three coders (LB-E, FF and NA). Factors operating at the ambulance service level-the organisation and the emergency and urgent care system in which it operated-were analysed by EK and LB-E.

EK read extracts of transcripts within a theme for each ambulance service separately. She analysed the theme, noting any variation in perceptions within each ambulance service, and discussing findings with LB-E and AO'C.
This was repeated for further themes, considering the connections between themes and refining the thematic framework by collapsing some themes. For example, themes around 'collaborative working' and 'the emergency and urgent care system' were merged because they were highly related. Each ambulance service was treated as a case and views of interviewees within each ambulance service were analysed together for each theme and subtheme. This part of the analysis focused on variation in how interviewees discussed non-conveyance related to their own ambulance service. This variation was explored qualitatively and is reported here.

\section{Ethical approval and consent}

All interviewees gave written informed consent (sent by email or fax prior to the interview). Approval was obtained from the National Research Ethics Service Committee North West, Greater Manchester West (REC reference 14/NW/1388). Direct quotes are used and labelled by ambulance service A-J to maintain anonymity. Job descriptions are not included in quote descriptors because of the small number of senior managers and commissioners interviewed for each ambulance service.

\section{Patient and public involvement}

The School of Health \& Related Research (ScHARR) at the University of Sheffield has a large portfolio of emergency and urgent care research projects. In order to support patient and public involvement (PPI) activities across these projects, a PPI group, the Sheffield Emergency Care Forum (SECF), has been established. This group provides direct support to research teams within ScHARR and also provides wider links to related PPI groups within the Yorkshire and Humber Clinical Research Network and Healthwatch.

Members of the SECF contributed to the study: Maggie Marsh attended the management meetings during the project and Enid Hirst provided input at the Project Advisory Group meetings.

In addition to having PPI members as part of the project team and Project Advisory Group, patients and members of the public working with three ambulance services or research teams engaged with emergency and urgent care came together to form a VAN project PPI group.

During the study, the PPI representatives were available to influence decisions being made about how to operationalise the research proposal and interpret findings. The PPI group took a lead in writing the plain English summary for the study and assisted in the construction of a wide reaching dissemination strategy.

\section{RESULTS}

\section{Recruitment}

We formally invited 80 individuals to interview. Most of those approached consented, with the exception of paramedics where 44 were approached but only 18 agreed to be interviewed. All 50 planned interviews were completed. Between four and seven interviews were undertaken in 
each ambulance service. The digital recording of one interview (a paramedic) was of poor quality and could not be transcribed and therefore 49 of the interviews undertaken were analysed.

\section{Overview of themes}

There were three broad themes related to how each ambulance service addressed non-conveyance: senior management commitment to non-conveyance, the ambulance workforce and the wider emergency and urgent care system in which the ambulance service was geographically located. Approaches to discharge at scene, which accounts for most non-conveyance, and approaches to ending calls with telephone advice only could differ within ambulance services and are discussed separately below.

\section{Senior management commitment to non-conveyance Motivation for non-conveyance: opportunity or risk}

Senior management in some ambulance services described non-conveyance as an opportunity to offer the most appropriate care to patients and as embedded practice within their organisations. In one organisation (Service C), non-conveyance was described as an historically important activity and there was an ongoing goal to improve or sustain high non-conveyance rates. Interviewees in both managerial and paramedic roles in this organisation described how they took a 'whole organisation' approach to non-conveyance and increasing rates of non-conveyance:

I think we've had that culture a lot longer [than other ambulance services. Staff [here] almost a decade ago were leaving people at home. Whereas in some services their non-conveyance rates are awful and I think their staff have still got to get their head around that's what their job is'. (Service C, interviewee 4)

In contrast, interviewees in some ambulance services described non-conveyance as an activity that had been prioritised in recent times (Service I) or was seen as less of a priority (Services B, E, F and H). At an extreme, senior management in one service was described as risk averse to non-conveyance. Senior management interviewees did not want to increase discharge at scene rates because of the risks involved to ambulance crews if something went wrong. The healthcare commissioner also viewed non-conveyance as a low priority within this ambulance service and a paramedic described this activity as a 'new concept'.

the [healthcare commissioners] and the GPs, and everybody else hate us, because, we, I won't release the crews to just leave people at home and make a call to a GP receptionist to say I've left this person at home because that crew member themselves doesn't realise what he's doing there, he's putting himself in the coroner's court in a non-defendable position, unless it's a safe structured handover recorded, and shared, I won't let it happen. The fact that it does happen all over the place is another matter and when I go round other ambulance services, I do think that this point is sometimes missed. (Service F, Interviewee 39)

In two services (Services $\mathrm{C}$ and $\mathrm{H}$ ), there appeared to be high levels of motivation to undertake clinical telephone assessment to manage demand for ambulances. In both services, there was a perception that services had a strategic vision for telephone assessment and provided workforce investment to support this:

Invested heavily [in the clinical hub] high level of expertise...invested a lot of time in that group of staff... we've put quite a large amount of investment into the structure. For us it's not just pulling paramedics off the road and sticking them in the control and getting them to [take] calls. We've got a whole [telephone advice] strategy here... we really thought through the whole thing. (Service H, Interviewee 49)

This contrasted with other services (Services A, D, $\mathrm{E}$ and $\mathrm{G}$ ) where interviewees perceived risk linked to the non-visual aspect of assessment, the use of paramedics rather than nurses (experienced in telephone triage) to undertake this role, and a potential risk to the organisation in not meeting patient expectations:

with [clinical telephone advice] ... what's your appetite for dealing with a high level of complaints... very simply, the more non-conveyance you do the [more] dissatisfaction you will have. People didn't phone 999 for a chat, they phoned 999 with an expectation that an ambulance was going to appear. (Service E, Interviewee 32)

\section{Structural stability}

The stability of the organisation was described differently for each ambulance service at the time of the interviews. Most ambulance services appeared to be stable, with no discussion of significant restructuring within their senior management. However, interviewees in four services (Services A, H, I and J) reported current or recent challenges in terms of loss of key senior personnel, or poor outcomes in a national quality assessment, which created challenges for senior management within the organisation. Interviewees did not expand explicitly on how this might impact on non-conveyance rates but it was clear from their discussions that their focus was on their key priority of meeting response time targets in the context of this instability rather than the innovation necessary for increasing non-conveyance rates:

it's been a difficult year because we were in such a difficult situation. We were very much around meeting [response time targets] and all of our focus was around increasing [staff] numbers and getting the response target right in the first instance, we were not necessarily entertaining anything that took paramedics away from core frontline business. (Service A, interviewee 5) 


\section{Ambulance workforce}

\section{Skill mix configuration}

Interviewees described how the configuration of their ambulance crews could affect discharge at scene rates. Paramedics with extended skills were viewed as having more confidence to discharge patients at scene than paramedics with standard training and paramedics were described as having more confidence than crews without paramedics. This highlighted the potential for the skill mix configuration within an ambulance service to affect its discharge at scene rate:

we've been particularly challenged by a shortage of senior clinicians, that is, paramedics. Paramedic numbers have been very low, and that will affect the decision-making of the clinicians and their confidence level. The more senior clinician you get on [discharge at scene] the more confidence they'll have to make a decision not to convey, whereas if you're running a number of non-paramedic based ambulances, so emergency medical technicians, or student ambulance paramedics, or emergency care assistants, generally the lower the grade the more likely the conveyance rates will be affected. (Service A, interviewee 5)

A similar variation in views was apparent for the provision of telephone assessment within the ambulance services. Many interviewees perceived telephone assessment to be a specialism, beyond the basic training of paramedics. They described the success of their clinical hub being underpinned by the support of specially trained paramedics and other clinicians such as mental health specialists (Services B, C and H). In contrast, in other services (Services A, E and G) interviewees described resource challenges within their service which appeared to limit the utilisation of multidisciplinary teams or senior paramedics within the clinical hub:

we don't have the resources to do hear and treat effectively...depleted in staffing, it's not supported enough to be able to work it effectively, and they're overwhelmed with work ...so, I would say the setup here is just under resourced, massively under resourced. (Service A, interviewee 17)

\section{Perceived value of higher skill mix}

The perceived value of paramedics with extended skills, for example, Emergency Care Practitioners, was described differently by interviewees in each ambulance service. In some ambulance services, there was consensus among the interviewees that paramedics with extended skills were established within the service and were perceived to be a valued resource for reducing conveyance to hospital (Services C, D and G). In these ambulance services, paramedics discussed being able to access their senior colleagues-paramedics with extended skills-and refer patients to them, also describing them as a support mechanism for seeking advice to facilitate their own decision-making regarding non-conveyance. Interviewees in these ambulance services wanted to expand this part of the workforce because of its perceived value to non-conveyance. In contrast, interviewees in other ambulance services described limited implementation of paramedics with extended skills, or a recent renewed interest in them, which implied that they had possibly been neglected within the organisation in the past (Services A, $\mathrm{E}, \mathrm{H}$ and $\mathrm{J}$ ). Reasons given for limited implementation of paramedics with extended skills included a need to focus resources elsewhere in the workforce or a lack of interest at a senior management level:

It's a bit of a 'dying on the vine' situation. Yes we have [paramedics with extended skills], a small number that were from the prerequisite arrangements. I'll never get rid of them, but we haven't trained [them] here [for a number of years]'. (Service E, interviewee 32)

\section{Organisational support for the workforce}

While many interviewees described how ambulance crews followed protocols and policies when making non-conveyance decisions, they also highlighted the importance of the confidence of crew members when deciding to discharge at scene. Indeed, one interviewee identified paramedic confidence as the most important factor affecting the decision to discharge patients at scene. Interviewees described how confidence could be improved through experience in the role, training to extend skills, mentoring in non-conveyance, the ability to discuss potential cases for non-conveyance with clinicians in the clinical hub, and being part of an organisation that was supportive of their workforce. Senior managers described the importance of supporting their workforce and understanding that a decision not to convey could be the right decision at the time even if it appeared not to be at a later stage. In contrast with the views of these senior managers, paramedics in some services openly described a fear of retribution if things went wrong (Services B, D, E, G, $\mathrm{H}$ and $\mathrm{J}$ ). Indeed, in some ambulance services, the managers described how supportive they were of the workforce when things go wrong but the paramedics interviewed expressed the opposite view (Services E, G, H and J).

probably the most important one is the support for staff. If we're encouraging the staff to non-convey it's making sure we're supporting them in that decision and if it does go wrong, making sure again that we understand the reason why it's gone wrong, but again continue to support the staff because they've probably made the right decision in the first place. (Service $\mathrm{G}$, interviewee 30 )

sadly, if something goes wrong out on the road, then obviously knowledge might get back to the station and it's talked about. And you're sadly in this job, you are known for being bad rather than being a good clinician. (Service G, interviewee 29) 


\section{The wider emergency and urgent care system}

Provision of alternative services to hospital

A consistent issue raised by interviewees from all ambulance services was the importance of having health and social care services available in the wider emergency and urgent care system to facilitate non-conveyance. Ambulance crews could work with these services to obtain information about patients to help them make a decision about non-conveyance or refer patients to those services to allow non-conveyance:

there's lots of expectation on ambulance services to single-handedly reduce conveyance to [an emergency department] but a lot of that, the success, is down to the buy-in from the local health community in terms, if they've not got the alternative pathway we can't use it. (Service C, interviewee 4)

There was variability in how interviewees described the provision of services that could enable non-conveyance. In one service (Service C), interviewees spoke positively about provision and the range of pathways available to the ambulance service. In contrast, interviewees in two services (Services A and I) spoke about a significant lack of pathways. Most other services (Services B, D, E, F, G, $\mathrm{H}$ and $\mathrm{J}$ ) described variation in provision across their region:

It's fair to say, very few options available to us as referral pathways. (Service A, interviewee 15)

Some areas have got really good pathways, so where you've got direct access to GPs, minor injuries units, walk in [centres], admission to certain [hospital] wards for certain clinicians. But then in other areas, actually the only place you've got is the emergency department, so the GPs won't take a referral from the paramedics [...] you'll get a minor injuries unit, often called a walk-in centre, and often the reason why we call it a walk-in centre is they don't take stretcher patients. So they have a perception that if you're an ambulance, the patient must be on a stretcher, therefore, they won't accept you. (Service D, interviewee 20)

\section{Connectivity with the wider system}

While ambulance services had no control over the availability of health and social care provision in their region, they are able to exert some influence over their engagement with these services. Interviewees in one service (Service C) shared consistently positive views about the connections between their ambulance service and services in the wider emergency and urgent care system. They described an initiative that linked ambulance crews with other services necessary for non-conveyance rather than the crews having to locate and contact these services themselves. This initiative was perceived as successful and to operate differently from other models across the country because it took some of the responsibility for discharge at scene off the ambulance crew. In contrast, another service described a similar initiative as a barrier to non-conveyance because it was not consistently provided across their region and thus caused more, rather than less, work for ambulance crews because they travelled across different localities with different processes and considerable efforts were required to identify the appropriate processes when wanting to non-convey.

some [models in other ambulance services] don't clinically take on the patient. They just say 'oh you need to phone such and such and refer them there'. With our [model] you basically hand [the patient] over and one of their clinicians [will] take responsibility for the patient $[\ldots]$ and they sort everything else out behind the scenes. (Service C, interviewee 4)

each one of the [Clinical Commissioning Groups] puts in what they call an SPA, a single point of access. Some of them put in several single points of access, which in itself is a bit of an oxymoron because it's not single point, it's several points of access, and so what we've done is then created another obstacle that a crew has to navigate before they can pass this job on. (Service B, interviewee 43)

In other services (Services A, B, D, E, F and I), interviewees perceived that relationships with key services in the wider system were poor because they could not access some services that might support non-conveyance, could not access services reliably when needed in the short time frame of needing to make non-conveyance decisions or were not confident in the quality of the service they needed to refer patients to. These services included primary care-both in or out of hours-community services, falls services, minor injury units and urgent care centres.

\section{Complexity of the system}

Interviewees described some ambulance services as situated within more complex emergency and urgent care systems than others. Clinical Commissioning Groups commission services for the population in their geographical locality. One ambulance service had 12 of these localities within their region whereas another had 33. Ambulance service managers described how each locality could have different priorities in terms of commissioning non-conveyance, creating complexity in terms of a single ambulance service having to deal with multiple commissioners' needs. Further complexity was highlighted by managers and paramedics who described how each Clinical Commissioning Group locality had different services operating in different ways. Understanding this variation between geographical areas within their region, or within the same service over time, created a burden for crew members who would decide to convey to an emergency department because this was the easiest option.

[the crew] go to a [patient who has had a] fall in [PLACE A], and [the crew] might be very familiar with the falls service that's back in [PLACE B], but 
now they're in [PLACE A] it's all completely different, the criteria's different, they haven't a clue, so they're left with the decision 'well, I haven't a clue what's going on here so do you know what, I'm just going to take them to the [emergency department]. (Service B, interviewee 43)

\section{DISCUSSION}

\section{Main findings}

There were some clear differences in interviewees' perceptions of how non-conveyance was addressed in different ambulance services. First, ambulance service senior management appeared to set the culture around non-conveyance within an organisation, viewing it either as an opportunity or as a risky endeavour. Although motivation levels to undertake non-conveyance did not appear to be directly affected by the stability of an ambulance service in terms of continuity of leadership and externally assessed quality, this stability could affect the ability of the organisation to innovate to increase non-conveyance rates. Second, descriptions of workforce configuration differed between ambulances services, as well as how this workforce was used, trained and valued. Third, interviewees in each ambulance service described health and social care in the wider emergency and urgent care system differently in terms of availability, amount of collaborative working with the ambulance service and complexity related to the numbers of services and healthcare commissioners with whom they had to work.

\section{Strengths and limitations}

The study took a national focus and is one of the first studies of variation in organisational perspectives of non-conveyance. Perspectives were gained from a breadth of stakeholders ranging from paramedics to senior management and from a stakeholder external to each organisationthe healthcare commissioner. A further strength is the inclusion of PPI representatives, from different parts of the country, offering insight into a study about staff and organisations, rather than patients. The study had two limitations. First, we recruited a small number of interviewees from each ambulance service, who may not share the same perceptions as their colleagues. We were mindful not to be swayed by the views of one individual within a service during the analysis and considered differing views expressed within an ambulance service. Second, the identification of paramedic participants was undertaken by managers in each ambulance service. This could have resulted in bias, with managers selecting paramedics who they perceived to have favourable views about their organisation. On reflection, we felt that interviewees gave open responses, expressing both positive and negative views about non-conveyance within their organisations.

\section{How does this fit with existing evidence?}

There has been little study of organisational perspectives of non-conveyance. Our findings are similar to those identified in a national audit of ambulance non-conveyance in England which included analysis of quantitative data on processes and outcomes and discussions with managers with each ambulance service. ${ }^{13}$ In particular, the audit found that while ambulance services have made advances in delivering non-conveyance, they may be limited by the increasingly complex health system in which they are operating within. For example, variation in the availability of alternative services in the wider emergency and urgent care system which the patient could be directed to, therefore avoiding transport to hospital, and the inevitable challenges of working with a large number of stakeholders operating within local health economies. Both of these factors were evident in the research reported in this article.

This paper focused on organisational factors in relation to ambulance non-conveyance. Other studies have taken a patient or clinician perspective to ambulance non-conveyance. Patients, accessing the ambulance service, can obtain the reassurance they look for ${ }^{14}$ and value the responsiveness and accessibility ${ }^{15}$ whether the outcome is conveyance or non-conveyance. Non-conveyance in terms of discharge at scene ${ }^{16}$ and telephone triage ${ }^{17}$ has been shown to be acceptable to service users and to carers. ${ }^{18}$ A number of papers report the complexity of decision-making among ambulance crews, balancing patient safety with patient choice. ${ }^{19-21}$ The variation reported in our paper may add a further layer of complexity for some ambulance crews making non-conveyance decisions. In addition, evidence shows that ambulance non-conveyance practices may reduce pressure in other parts of the emergency care system by reducing emergency department attendances ${ }^{16}$ and hospital admission rates. ${ }^{6}$ However, our work has shown that the relationships between the ambulance service and other parts of the emergency and urgent care system, required to facilitate non-conveyance, can be complicated often due to the size of ambulance service regions and a lack of consistency in service provision across each region. This fits with recent evidence of the challenges faced by ambulance services which need to collaborate with large numbers of stakeholders across the geographical area they serve ${ }^{13}$ and that of other authors who have identified a lack of appropriate alternative services as a barrier to facilitating ambulance non-conveyance. ${ }^{19} 2022$ Interviewees in our study felt that the skill mix of the workforce and the associated confidence perceived in more highly skilled staff was important in optimising non-conveyance. This is supported by other evidence showing that advanced paramedics/paramedics with extended skills can achieve higher non-conveyance rates than paramedics. ${ }^{10}$

\section{Implications}

Healthcare policy in England supports the use of clinically appropriate non-conveyance, providing care closer to home for patients ${ }^{5}$ and in doing so reducing pressure within emergency departments and the wider emergency care system. Standardising practices between ambulance services is a key focus of this policy. National policy directives will influence regional ambulance services, 
particularly those making strategic service level decisions. While national policy drivers were discussed by our interviewees, there was little variation in views between ambulance services, and therefore discussion about national policy is not reported here.

To some extent, ambulance services have the autonomy to increase non-conveyance in terms of the workforce they employ, and how they use them within the service. Importantly, however, there is a degree of reliance on other providers within the emergency and urgent care system to facilitate some forms of ambulance non-conveyance. This requires collaborative working to create and maintain, safe and appropriate referral pathways which support non-conveyance.

The findings of the study reported here were used in a statistical regression to identify factors explaining variation in rates of discharge at scene and telephone advice between ambulance services in England..$^{23}$ Variation in discharge at scene rates was explained by differences in managerial motivation and workforce configuration and value, but not by the complexity of the wider emergency and urgent care system.

The focus of this paper was to assess NHS workforce views. The variation we found in organisational approaches to non-conveyance may impact on patient and carer experience. Further research into understanding how variation in ambulance non-conveyance impacts on the patient/carer experience is desirable.

\section{CONCLUSION}

Health policy in England encourages use of initiatives to provide care closer to home, one of which is clinically appropriate non-conveyance following a 999 ambulance call. There is considerable variation in non-conveyance rates across the 10 regional ambulance services in England. This study suggests that factors within and outside the control of ambulance services may contribute to variation in non-conveyance rates. Aspects within the control of ambulance services include motivation of senior management, workforce configuration and value placed on senior clinical skill mix. Aspects outside the control of ambulance services include the complexity of the emergency and urgent care systems they operate within, although to some extent, the collaborations they build with services with these systems is within their control.

Acknowledgements We would like to acknowledge the following: Richard Campbell for transcribing the interview recordings; Flavia Fernandes and Nisar Ahmed for their assistance with the coding of the interview data and Enid Hirst and Maggie Marsh for their contribution to the study PPI groups.

Contributors EK and $A 0^{\prime} C$ designed the study and wrote the first draft of the paper. LB-E collected the data. EK led, and along with LB-E undertook the analysis of the data with advice from AO'C. All authors commented on drafts and agreed the final version.

Funding This work was supported by NIHR (Health Service and Delivery Research Programme 13/54/75). The views expressed are those of the authors and not necessarily those of the NHS, the NIHR or the Department of Health.
Competing interests None declared.

Patient consent Not required.

Ethics approval National Research Ethics Service Committee North West, Greater Manchester West (REC reference 14/NW/1388).

Provenance and peer review Not commissioned; externally peer reviewed.

Data sharing statement The risk of breaking anonymity is too high to share this data.

Author note The COREQ reporting statement was completed.

Open access This is an open access article distributed in accordance with the Creative Commons Attribution Non Commercial (CC BY-NC 4.0) license, which permits others to distribute, remix, adapt, build upon this work non-commercially, and license their derivative works on different terms, provided the original work is properly cited, appropriate credit is given, any changes made indicated, and the use is non-commercial. See: http://creativecommons.org/licenses/by-nc/4.0/.

\section{REFERENCES}

1. NHS England. Ambulance quality indicators. https://www.england. nhs.uk/statistics/statistical-work-areas/ambulance-quality-indicators/ (Accessed 14 May 2018).

2. Hoikka M, Silfvast T, Ala-Kokko TI. A high proportion of prehospital emergency patients are not transported by ambulance: a retrospective cohort study in Northern Finland. Acta Anaesthesio Scand 2017;61:549-56.

3. Carrigan S. The prevalence and characteristics of non-conveyanced patients in Nova Scotia, 2016.

4. Snooks HA, Dale J, Hartley-Sharpe C, et al. On-scene alternatives for emergency ambulance crews attending patients who do not need to travel to the accident and emergency department: a review of the literature. Emerg Med J 2004;21:212-5.

5. NHS England. Transforming urgent and emergency care services in England. Clinical models for ambulance services London: Urgent and Emergency Care Review programme team, 2015.

6. O'Cathain A, Knowles E, Maheswaran R, et al. A system-wide approach to explaining variation in potentially avoidable emergency admissions: national ecological study. BMJ Qual Saf 2014;23.

7. Ebben RHA, Vloet LCM, Speijers RF, et al. A patient-safety and professional perspective on non-conveyance in ambulance care: a systematic review. Scand J Trauma Resusc Emerg Med 2017;25:71.

8. O'Cathain A, Knowles E, Bishop-Edwards L, et al. Understanding variation in ambulance service non-conveyance rates: a mixed methods study. Health Serv Deliv Res 2018;6.

9. Office for National Statistics. Clinical Commissioning Groups (April 2018) names and codes in England. 2018. http://geoportal.statistics. gov.uk/datasets/4010cd6fc6ce42c29581c4654618e294_0 (Accessed 14 May 2018).

10. Tohira H, Williams TA, Jacobs I, et al. The impact of new prehospital practitioners on ambulance transportation to the emergency department: a systematic review and meta-analysis. Emerg Med J 2014;31:e88-e94.

11. QSR International Pty Ltd. NVivo qualitative data analysis software. 2012. Version 10.

12. Ritchie J, Spencer L. Qualitative data analysis for applied policy research. The qualitative researcher's companion 2002;573:305-29.

13. National Audit Office. NHS ambulance service. London: National Audit Office, 2017.

14. Togher FJ, O'Cathain A, Phung VH, et al. Reassurance as a key outcome valued by emergency ambulance service users: a qualitative interview study. Health Expect 2015;18:2951-61.

15. Booker MJ, Simmonds RL, Purdy S. Patients who call emergency ambulances for primary care problems: a qualitative study of the decision-making process. Emerg Med J 2014;31:448-52.

16. Mason S, Knowles E, Colwell B, et al. Effectiveness of paramedic practitioners in attending 999 calls from elderly people in the community: cluster randomised controlled trial. BMJ 2007;335:919.

17. Care Quality Commission. Ambulance survey of 'Hear and Treat' callers 2013/14. 2017. http://www.cqc.org.uk/publications/surveys/ ambulance-survey-hear-treat-callers-201314 (Accessed 14 May 2018).

18. Knowles E, Mason S, Colwell B. An initiative to provide emergency healthcare for older people in the community: the impact on carers. Emerg Med J 2011;28.

19. Burrell L, Noble A, Ridsdale L. Decision-making by ambulance clinicians in London when managing patients with epilepsy: a qualitative study. Emerg Med J 2013;30. 
20. O'Hara R, Johnson M, Siriwardena AN, et al. A qualitative study of systemic influences on paramedic decision making: care transitions and patient safety. J Health Serv Res Policy 2015;20(1 Suppl):45-53.

21. Porter A, Snooks H, Youren A, et al. Should I stay or should I go?' Deciding whether to go to hospital after a 999 call. J Health Serv Res Policy 2007;12:32-8.
22. Noble AJ, Snape D, Goodacre S, et al. Qualitative study of paramedics' experiences of managing seizures: a national perspective from England. BMJ Open 2016;6:e014022.

23. O'Cathain $A$, Jacques $R$, Stone $T$, et al. Why do ambulance services have different non-transport rates? A national cross sectional study. PLoS One 2018;13:e0204508. 